\title{
An Electric Spring Control Strategy Based on Finite Control Set-Model Predictive Control
}

\author{
Tao Zhang*, Qiang Hao, Zheng Zheng, Chuang Lu \\ School of Electrical Engineering and Automation, Henan Polytechnic University, Jiaozuo 454003, China
}

Corresponding Author Email: zhangtao@ @pu.edu.cn

https://doi.org/10.18280/jesa.530403

Received: 25 April 2020

Accepted: 19 June 2020

\section{Keywords:}

electric spring (ES), finite control set-model predictive control (FCS-MPC), voltage fluctuation, power quality

\begin{abstract}
As a novel voltage control device, electric spring (ES) can effectively suppress the voltage fluctuations across critical loads (CLs), and solve the various problems with electrical quality induced by the grid access of renewable energy resources (RES). However, the traditional controllers for the ES system can no longer meet the control requirements, as the environment is complicated by the growing number of load-side nonlinear loads and uncertain disturbances. To solve the problem, this paper proposes a control system based on finite control set-model predictive control (FCS-MPC), and applies it to the ES. Firstly, a load-side circuit prediction model was established and analyzed. Next, a control system was designed based on FCS-MPC. Finally, the proposed system was proved feasible and effective through MATLAB/Simulink simulation and dSPACE physical experiment. The results show that the proposed FCS-MPC system can directly control the ES, easily handle system constraints, achieve robust dynamic and static performance, eliminating the need for pulse width modulation (PWM).
\end{abstract}

\section{INTRODUCTION}

The development of renewable energy sources (RES) has accelerated the reform of the energy structure. However, the intermittency and unsustainability of RES have brought various power quality problems to the power supply and distribution network [1, 2]. Proposed by Hui et al. [3], electric spring (ES) provides a new solution to such problems. Unlike the traditional circuit compensation device, the ES operates in a distributed manner on the load side, making the power consumption more reliable on the load side [4]. Compared with the static var compensator (SVC), the ES boasts small capacity and high reliability [5]. Different from the traditional measures to alleviate RES supply fluctuations (e.g. controlling load switches), the ES improves the comfort and happiness of power users, without hindering the normal power consumption on the load side [6].

The original ES structure can only realize reactive power compensation: the device is connected in series with the noncritical loads (NCLs), which are insensitive to voltage changes, forming smart loads (SLs); then, the ES transfers the voltage fluctuations on the bus to the NCLs, thereby stabilizing the bus voltage. In this way, the voltage of the critical loads (CLs) on the bus, which are sensitive to voltage changes, will also be stabilized. Through years of research, the ES has been proved to be feasible in frequency stabilization, power factor correction, and filtering $[7,8]$.

Over the years, the ES control has become increasingly robust, nonlinear, and intelligent. The control devices have evolved from the proportional-integral (PI) controller suitable for ES-1 topology [9, 10], to quasi-proportional resonant (PR) controller for ES-2 topology [11], and to intelligent controllers like fuzzy controller [12]. The increase of nonlinear loads, coupled with various circuit interferences, raise stricter requirements on the normal operation of the ES. In alternative current (AC) ES control system, the PI controller cannot achieve error-free tracking of the AC reference signal, and the parameter debugging of the traditional linear controller is time-consuming and laborious. To solve the problem, more and more nonlinear control theories have been proposed and applied to inverter systems, such as hysteresis control, sliding mode control, repetitive control, and adaptive control [13].

Model predictive control (MPC) is an algorithm that uses the system model to predict the controlled quantity and approximate it to the reference quantity [14]. The algorithm supports fast dynamic response and transient response, and adapts well to nonlinear constraints. However, the MPC theory was not widely accepted at the beginning, because the control system needs a high-performance hardware platform to perform the numerous calculations in each sampling period. Thanks to the development of computer technology, the MPC has become a research hotspot in the control of power electronic converters $[15,16]$. For instance, the finite control set-model predictive control (FCS-MPC) $[17,18]$ can achieve flexible and multivariable control, eliminating the need for pulse width modulation (PWM) of the output. It is suitable for nonlinear systems with constraints. Previous studies $[19,20]$ have confirmed the feasibility of the MPC in the control of single-phase and three-phase grid-connected inverters. Unlike other control methods, the MPC takes the predicted circuit quantities in future as the reference for the current actions of the system.

This paper introduces the FCS-MPC into ES control system, with the aim to improve the anti-interference ability of the ES and to optimize the dynamic and static ES performance under complex load environment. Firstly, a load-side circuit prediction model was established and analyzed. On this basis, the prediction formula of ES output voltage and the calculation formula of ES reference formula were created, as well as the performance indicator function. Finally, the proposed control 
strategy was proved feasible and effective through MATLAB simulations and dSPACE physical experiments, and the simulation and experimental results were given in the presence of interference in the circuit.

\section{MODELLING AND ANALYSIS OF ES SYSTEM}

Figure 1 shows the structure of the ESs connected to the power system, where $Z_{0}, Z_{1}$, and $Z_{2}$ are the impedances of the transmission line. On the supply side, there is a RES and a grid supply. On each load unit, there is an SL made up of an ES serial-connected with an NCL. Each SL is connected in parallel with the CL into a load unit. When the RES power supply fluctuates, the ESs of all load units on the line participate in the regulation. The failure of a single ES does not affect the stability of the bus voltage, providing a guarantee for the reliability of power consumption.

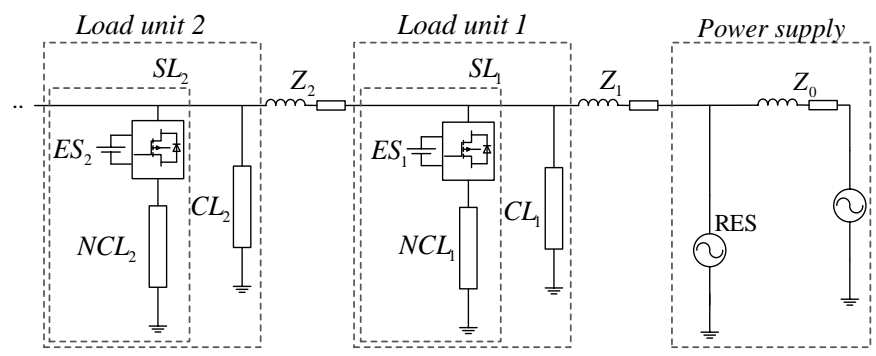

Figure 1. The structure of the ESs connected to the power system

The topologies and functions of ES-1 to ES-7 are introduced in details by Wang et al. [21]. Among them, ES-2 topology is selected for this research. ES-2 topology is extended from ES1 topology by adding a battery on the direct current (DC) side, achieving the new function of power consumption [22, 23].

Figure 2 is the simplified model of a single ES load unit and the supply side, where $U_{g}$ is the supply-side voltage, $Z_{t}$ is the transmission line impedance, $U_{t}$ is the transmission line voltage drop, $I_{t}$ is the bus current, $Z_{c l}$ and $Z_{n c l}$ are respectively the impedances of CL and NCL, and $U_{c l}$ and $U_{n c l}$ are respectively the voltages of CL and NCL.

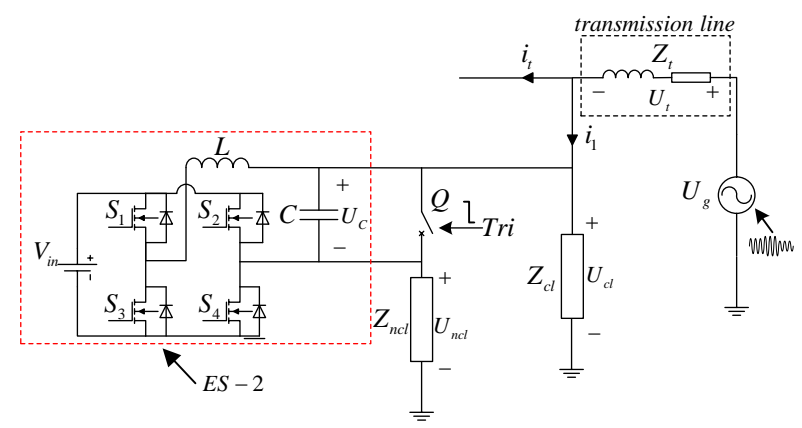

Figure 2. The topology of a single load unit

The ES-2 circuit topology is given in the red dotted box, where $S_{1} \sim S_{4}$ are switching tubes, $\mathrm{L}$ and $\mathrm{C}$ are respectively the inductance and capacitance of the low-pass filter, $U_{C}$ is the output voltage of ES, $V_{i n}$ is the battery voltage on the DC side of the inverter bridge, Q is the ES switch, and Tri is the switch signal.
When a compensation demand arises in the circuit, the Tri signal disconnects $\mathrm{Q}$, so that ES and NCL are connected in series. Then, the controller collects the circuit quantities, and calculate the control signal to act on $S_{1} \sim S_{4}$. Through the control, the DC is inverted to $\mathrm{AC}$, and the ES generates the output voltage, making the bus voltage stable. When the ES is needed for circuit regulation, Q is closed to short-circuit ES.

\section{ESTABLISHMENT OF CONTROL STRATEGY}

This paper constructs a control system based on the ES system model (Figure 3 ). The principle of the control system goes as follows: the CL reference voltage $U_{\text {ref }}$ is generated based on the CL voltage $U_{c l}$ collected by the voltage sensor, and imported to the MPC to output a control signal for switch tubes $S_{1} \sim S_{4}$ on the inverter bridge; through the control, the output voltage of the ES $U_{C}$ approaches the reference voltage $U_{C r e f}$, that is, the error between the two voltages is minimized.

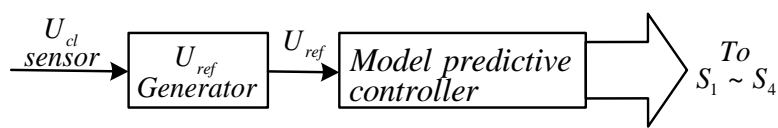

Figure 3. The block diagram of our control system

The performance indicator function, which is often adopted in prediction models, measures the optimization trend of system indices. In MPC rolling optimization, the function is the key player that decides the controller output, and the enabler of the MPC to solve multivariable constrained problems. To simplify the control structure and reduce the calculation amount of the control system, the square of the difference $g_{\min }$ between $U_{\text {Cref }}$ and the predicted value was selected as the performance indicator function:

$$
\mathrm{g}_{\text {min }}=\left(U_{\text {Cref }}(k+1)-\hat{U}_{C}(k+1)\right)^{2}
$$

The calculation of $g_{\min }$ depends heavily on three parameters: the CL reference voltage $U_{\text {ref }}$, the predicted ES output voltage $\widehat{U}_{C}(k+1)$, and the reference value of the output voltage $U_{C r e f}$. Therefore, the calculation process of the three parameters is detailed below.

\subsection{Generation of $U_{\text {ref }}$}

The $U_{\text {ref }}$ was generated by the method shown in Figure 4, for the stability of the amplitude and phase of CL voltage.

When ES is not needed for circuit regulation, the Swi signal is set to low level, and the Tri signal (Figure 2) to high level. Then, switch Q is closed, the voltage sensor collects $U_{c l}$, and the phase of $U_{c l}$ is obtained through the phase-locked loop. Next, channel 1 in the switch is selected for signal transmission, and the phase is passed through the sin module and booster $\mathrm{m}$ to generate the CL reference voltage $U_{r e f}$. But $U_{\text {ref }}$ has not effect at this time.

When ES is needed for circuit regulation, the Swi signal is set to high level, channel 2 in the switch is selected for signal transmission, and the Tri signal is set to low level. Then, switch Q is opened, and ES is connected in parallel to the bus. The phase signal of $U_{\text {ref }}$ will change periodically after passing 
the time delay module. The delay is an integer number of power frequency cycles.

The $U_{\text {ref }}$ generation method in Figure 4 keeps $U_{\text {ref }}$ unchanged regardless of circuit conditions, eliminates the interference of external factors on CL, and minimizes the dependence on external circuit parameters, thereby improving the robustness of our control system.

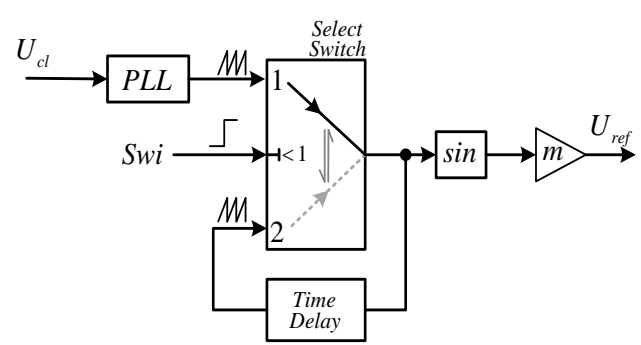

Figure 4. The structure of $U_{\text {ref }}$ generator

\subsection{Prediction of $\widehat{U}_{C}(k+1)$}

To analyze the load-side model, Figure 2 was further simplified in the ES working state into Figure 5, where $i_{1}$ is the current input to a single load unit on the bus, $i_{2}$ is the CL current, $U_{c l}$ is the CL voltage, $i_{3}$ is the SL current, $i_{L}$ and $i_{c}$ are respectively the inductance and capacitance currents of the filter, $U_{c}$ is the ES output voltage, $U_{n c l}$ is the NCL voltage, $u V_{i n}$ is the inverter bridge output voltage, with $\mathrm{u}$ being the switching function. If $\mathrm{u}=1, S_{1}$ and $S_{4}$ (Figure 2) are closed; if $\mathrm{u}=0, S_{1}$ and $S_{2}$ are closed, or $S_{3}$ and $S_{4}$ are closed; if u=-1, $S_{2}$ and $S_{3}$ are closed.

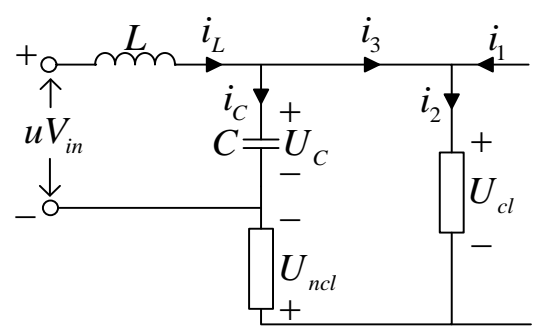

Figure 5. The simplified structure of the load side during ES operation

To reduce the influence of the sensor in the circuit on the predicted value, this paper only collects the inductor $L$ current and the capacitor $C$ voltage to predict the ES output voltage:

$$
\begin{aligned}
& L \frac{d i_{L}}{d t}=u V_{i n}-U_{C} \\
& C \frac{d U_{C}}{d t}=i_{L}-i_{3}
\end{aligned}
$$

Let $T_{s}$ be the discrete sampling period of the ES control system. From formula (2) and the forward difference formula [24], the current of the inductor $L$ at time $k$ can be predicted by:

$$
\hat{i}_{L}(k)=i_{L}(k-1)+\frac{T s}{L}\left(u V_{i n}(k)-U_{C}(k)\right)
$$

From formula (3) and backward difference formula [25], the ES output voltage at time $k+1$ can be predicted by:

$$
\hat{U}_{C}(k+1)=U_{C}(k)+\frac{T s}{C}\left(\hat{i}_{L}(k)-\hat{i}_{3}(k)\right)
$$

For convenience, it is assumed that $i_{3}$ does not change in a sampling period $T_{s}$ :

$$
\hat{i}_{3}(k) \approx \hat{i_{3}}(k-1)
$$

From formula (3), formula (6), and forward difference formula [24], we have:

$$
\hat{i_{3}}(k-1)=i_{L}(k-1)-\frac{C}{T s}\left(U_{C}(k)-U_{C}(k-1)\right)
$$

Substituting formulas (4), (6), and (7) into formula (5), we have:

$$
\hat{U}_{C}(k+1)=\frac{T s^{2}}{L C} u V_{i n}(k)+\left(2-\frac{T s^{2}}{L C}\right) U_{C}(k)-U_{C}(k-1)
$$

\subsection{Calculation of $\boldsymbol{U}_{\text {Cref }}$}

To eliminate the interference of bus current $i_{1}$ on the system, the output voltage control was adopted, with the controlled quantity being volage $U_{c}$. When the supply side fluctuates, the bus current changes accordingly. To stabilize $U_{c l}$, the amplitude and phase of ES output voltage will both change. The controlled quantity can be derived from the circuit in Figure 5:

$$
U_{C}=U_{c l}+U_{n c l}
$$

Substituting current $i_{l}$, and load parameters $Z_{n c l}$ and $Z_{c l}$ into formula (9), the reference value $U_{C r e f}$ of ES output voltage $U_{C}$ at time $\mathrm{k}+1$ can be expressed as:

$$
U_{\text {Cref }}(k+1)=\left(1+\frac{Z_{n c l}}{Z_{c l}}\right) U_{r e f}(k+1)-Z_{n c l} i_{1}(k+1)
$$

where, $U_{\text {ref }}$ is the reference voltage of CL, whose effective value remains constant; $i_{l}$ is the current flowing from the bus into the load side, which changes in real time under the effects of changes on both supply side and load side. $U_{\text {Cref }}$ also changes in real time.

Before calculating $U_{\text {Cref }}$ by formula (10), it is necessary to obtain the values of $i_{1}$ and $U_{r e f}$ at time $\mathrm{k}+1$. Since the change of $U_{r e f}$ is periodic, and $i_{l}$ is collected from the circuit, it is assumed that $i_{1}$ does not change between two adjacent sampling periods:

$$
i_{1}(k+1) \approx i_{1}(k)
$$

The $i_{l}$ value at time $\mathrm{k}$ was measured by installing a current sensor on the outside of the load unit. The calculation method of $i_{1}$ in formula (10) enhances the anti-interference ability of the control system, and optimizes the dynamic performance of 
the system under supply-side fluctuations.

\section{FCS-MPC CONTROL SYSTEM}

As shown in Figure 6, the FCS-MPC control system first derives $U_{\text {Cref }}(k+1)$ and $U_{C}$ from $U_{r e f}, i_{l}$, and circuit parameters. Then, the $\widehat{U}_{C}(k+1)$ value is predicted from these results and the switching function states $u$ of full-bridge inverter of the ES. Coupling the predicted result with the performance indicator function, the optimal value $u_{\text {opt }}$ is directly applied to generate the driving signal. The $u_{\text {opt }}$ does not need to be modulated.

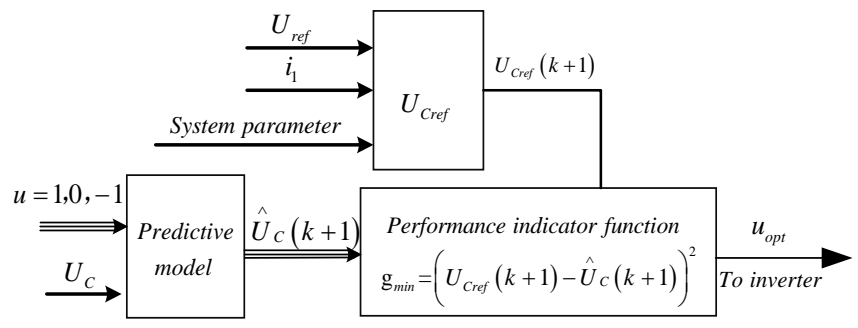

Figure 6. The block diagram of the FCS-MPC control system

In this paper, the ES full-bridge inverter (Figure 2) is regarded as a nonlinear discrete system with 4 different vectors. The gate signals $K_{1}$ and $K_{2}$ can be defined as:

$$
\begin{aligned}
& K_{1}= \begin{cases}1 & S_{1} \text { is opened and } S_{3} \text { is closed } \\
0 & S_{1} \text { is closed and } S_{3} \text { is opened }\end{cases} \\
& K_{2}= \begin{cases}1 & S_{2} \text { is opened and } S_{4} \text { is closed } \\
0 & S_{2} \text { is closed and } S_{4} \text { is opened } .\end{cases}
\end{aligned}
$$

The switch states and output voltages are shown in Table 1. The output voltage has three states: $+V_{i n}, 0$, and $-V_{i n}$. In each sampling period, the control system needs to calculate the values of the performance indicator function under the three states. Then, the $u$ value corresponding to the minimum value is taken as the controller output.

Table 1. The switch states and voltage vectors

\begin{tabular}{cccc}
\hline $\boldsymbol{K}_{\mathbf{1}}$ & $\boldsymbol{K}_{\mathbf{2}}$ & $\boldsymbol{u}$ & Output voltage \\
\hline 0 & 0 & 0 & 0 \\
1 & 0 & 1 & $+V_{\text {in }}$ \\
1 & 1 & 0 & 0 \\
0 & 1 & -1 & $-V_{\text {in }}$ \\
\hline
\end{tabular}

In each sampling period, the process in Figure 4 is implemented once. After the optimal output is obtained and transmitted to the inverter bridge, the sampling and calculation will be repeated in the next sampling period. Since the control system always looks for the switch state that makes the predicted value approach the reference value, $U_{c}$ continues to approximate $U_{c r e f}$, that is, $U_{c l}$ continues to approach $U_{r e f}$. This control method minimizes the influence of change of bus current $i_{l}$ over the load unit, and exhibits a certain antiinterference ability.

In ideal conditions, the entire control process, from sampling, calculation, decision-making to generating the output voltage, is completed in an instant, that is, the control time tends to infinitesimal. Figure 7 shows the variation of the controlled quantity with the reference value in ideal conditions. In the actual situation, however, it takes a certain time for the system to complete the control process. Figure 8 presents the variation of the controlled quantity with the reference value in the actual situation.

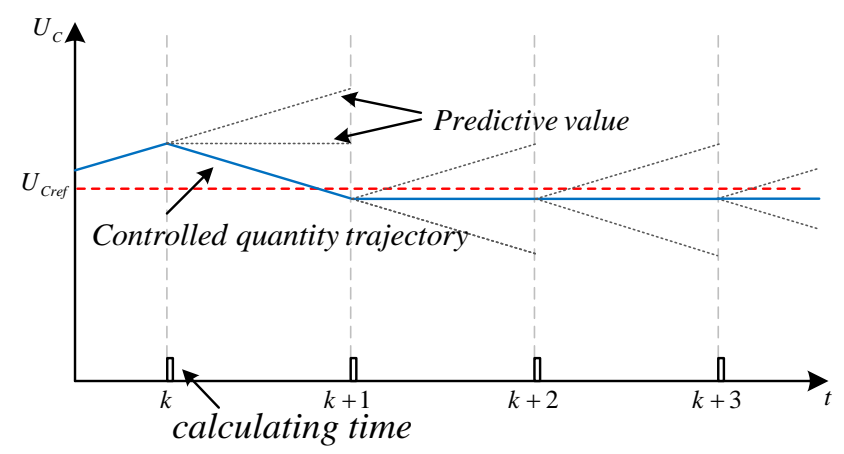

Figure 7. The trajectory of the controlled quantity in ideal conditions

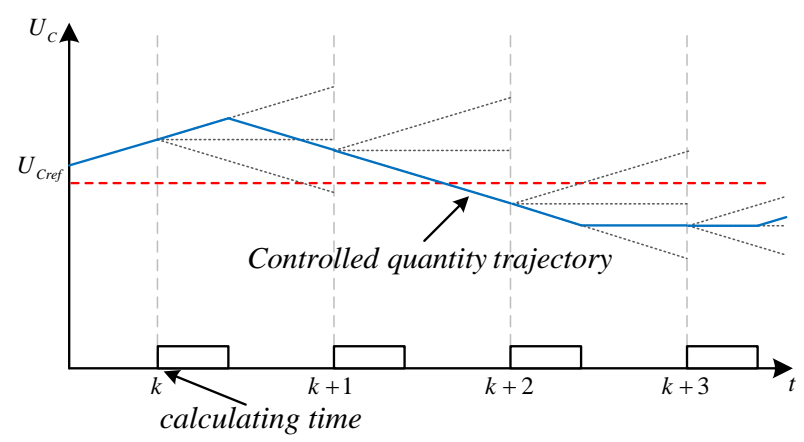

Figure 8. The trajectory of the controlled quantity in actual situation

As shown in Figure 7, sampling, calculation, decisionmaking, and control were completed simultaneously in ideal conditions, and the controlled quantity could be stabilized in the shortest possible time. As shown in Figure 8, the entire control process was delayed by a calculation cycle, because the calculation consumed a certain amount of time. In the actual situation, the controlled quantity oscillated near the reference value, making the system unstable. To mitigate the negative impact of calculation time, this paper simplifies the structure of the control system to the maximum extent. Therefore, the calculation-induced delay in our system can be neglected. Moreover, the calculation time of our system can fully meet the requirements on the dSPACE physical experiment platform.

\section{VERIFICATION}

\subsection{Simulation and result analysis}

To verify the proposed FCS-MPC control system for the ES, a simulation model was established on MATLAB/Simulink to simulate the control effect of the system. The simulation circuit was built in accordance with Figure 2. The circuit parameters and controller parameters are listed in Table 2. Considering the effects of the sampling period on the quality of the controlled quantity in discrete sampling, the simulation 
was carried out under different sampling periods: $2 \mathrm{e}-5 \mathrm{~s}, 1 \mathrm{e}-5 \mathrm{~s}$, and 1e-6s. The $U_{c l}$ values under these periods are compared in Figure 9.

Table 2. The hardware parameters of the simulation circuit

\begin{tabular}{cc}
\hline Parameter & Value \\
\hline Transmission line impedance Zt & $0.6 \Omega+2.86 \mathrm{mH}$ \\
CL Zcl & $40 \Omega$ \\
NCL Zncl & $4 \Omega$ \\
Filter inductance L & $3.6 \mathrm{mH}$ \\
Filter capacitance C & $100 \mu \mathrm{F}$ \\
Battery voltage Vin & $360 \mathrm{~V}$ \\
\hline
\end{tabular}

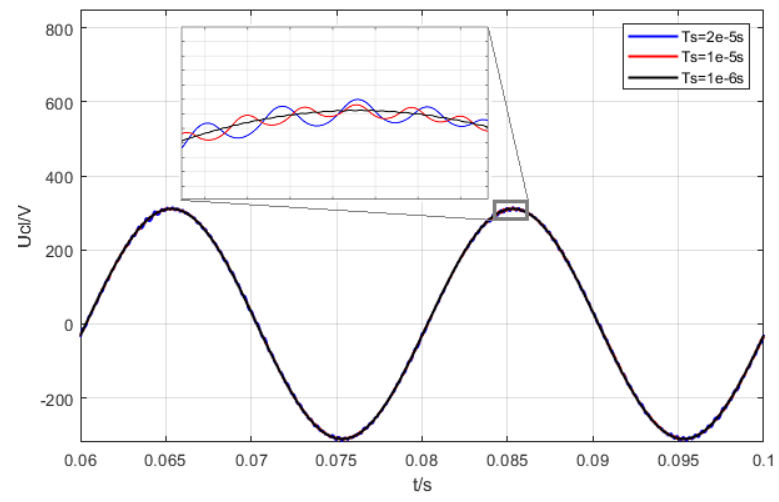

Figure 9. The comparison of $U_{c l}$ under different sampling periods

As shown in Figure 9, the shorter the sampling period, the smaller the fluctuation of the controlled quantity, and the closer the waveform is to the ideal sine. Because the MPC has no modulation link, the trigger pulse frequency it generates depends entirely on the discrete sampling period of the system. The shorter the sampling period, the higher the accuracy of the prediction result, and the faster the $\mathrm{CL}$ will enter the normal working environment in terms of electrical energy. Repeated simulations show that the proposed control system achieved fast calculation speeds under all three sampling periods, and experienced virtually no delay. As a result, the minimum sampling period of 1e-6s was adopted for subsequent simulation.

\subsubsection{Simulation at $220 \mathrm{~V}$}

The next step is to verify whether the ES in the FCS-MPC control system could stabilize the CL voltage quickly and stably. Hence, the amplitude and effective value of $U_{c l}$ were observed under the drop and rise of supply-side voltage, respectively. After debugging in MATLAB simulation, it is learned that the load-side load voltage is $220 \mathrm{~V}$, when the supply-side voltage $U_{g}$ is $262 \mathrm{~V}$, under the circuit parameters in Table 2 . Then, the supply-side voltage $U_{g}$ was decreased and increased by $10 \%$ to $235.8 \mathrm{~V}$ and $288.2 \mathrm{~V}$, respectively, and maintained for $0.1 \mathrm{~s}$. The simulation time was set to $0.4 \mathrm{~s}$. The time variations of $U_{g}$ and Tri signal are recorded in Table 3.

As shown in Table 3, the supply-side voltage remained normal in $0 \sim 0.2 \mathrm{~s}$, and resumed the normal state in $0.3 \sim 0.4 \mathrm{~s}$ after the voltage dropped or rose in $0.2 \sim 0.3 \mathrm{~s}$. Figures 10 and 11 present the variations in $U_{c l}$ amplitude during voltage drop and voltage rise, respectively. It can be seen that, when supply- side voltage fluctuated, the $U_{c l}$ amplitude in the circuit with ES remained basically unchanged in $0 \sim 0.5 \mathrm{~s}$, ensuring the stable and good power environment at both ends of $Z_{c l}$; the $U_{c l}$ amplitude in the circuit without ES fluctuated significantly in $0.2 \sim 0.3 \mathrm{~s}$.

Table 3. The time variations of $U_{g}$ and Tri signal

\begin{tabular}{ccc}
\hline Time/s & Ug/V & Tri \\
\hline $0 \sim 0.1$ & 262 & 1 \\
$0.1 \sim 0.2$ & 262 & 0 \\
$0.2 \sim 0.3$ & $235.8($ drop) $/ 288.2$ (rise) & 0 \\
$0.3 \sim 0.4$ & 262 & 0 \\
\hline
\end{tabular}

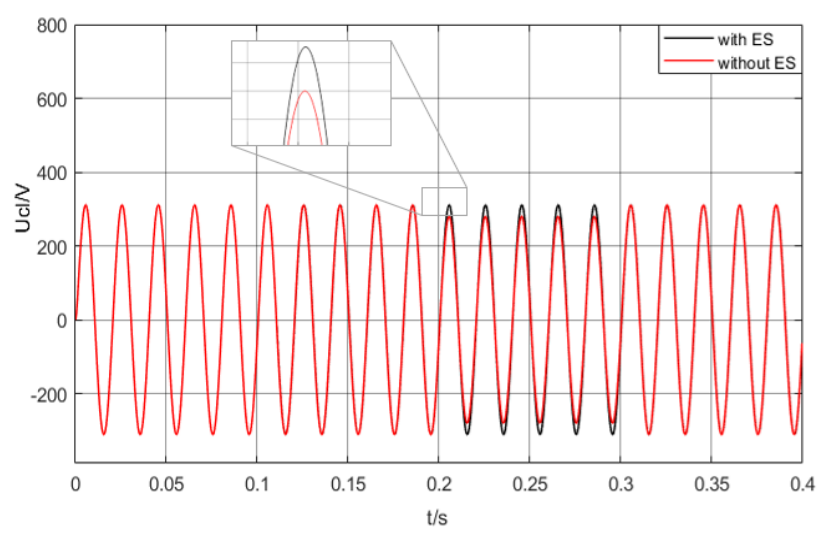

Figure 10. The $U_{c l}$ amplitude at the drop of supply-side voltage

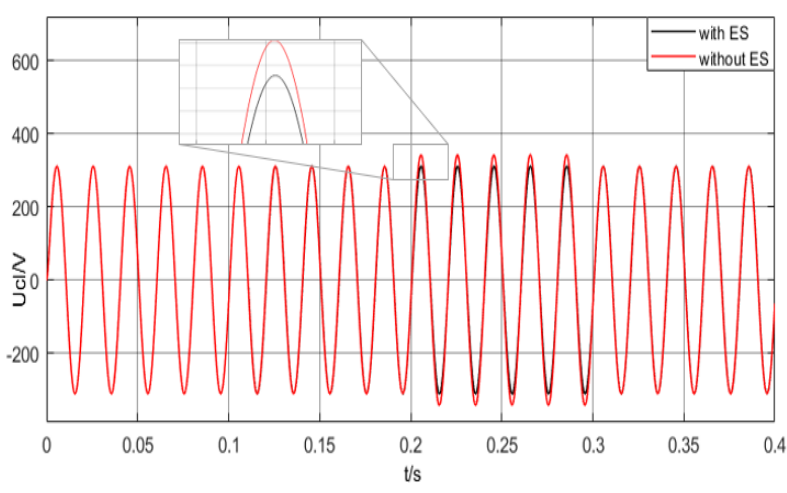

Figure 11. The $U_{c l}$ amplitude at the rise of supply-side voltage

Figures 12 and 13 display the variations in the effective value of $U_{c l}$ during voltage drop and voltage rise, respectively. It can be observed that the effective value of $U_{c l}$ in the circuit with ES fluctuated slightly after $0.1 \mathrm{~s}$, and then stabilized quickly at the reference value, owing to the sudden switching of the ES. When the supply-side voltage fluctuated, the $U_{c l}$ always remained stably at about $220 \mathrm{~V}$, regardless of the voltage drop or rise in the circuit with ES, providing a stable and reliable power environment for $Z_{c l}$. By contrast, the effective value of $U_{c l}$ in the circuit without ES fluctuated with the supply-side voltage. Overall, the ES under FCS-MPC performs excellently in stabilizing $U_{c l}$ voltage during voltage rise and voltage drop, which demonstrates the feasibility of the FCS-MPC control system and its strong adaptability to voltage fluctuations. 


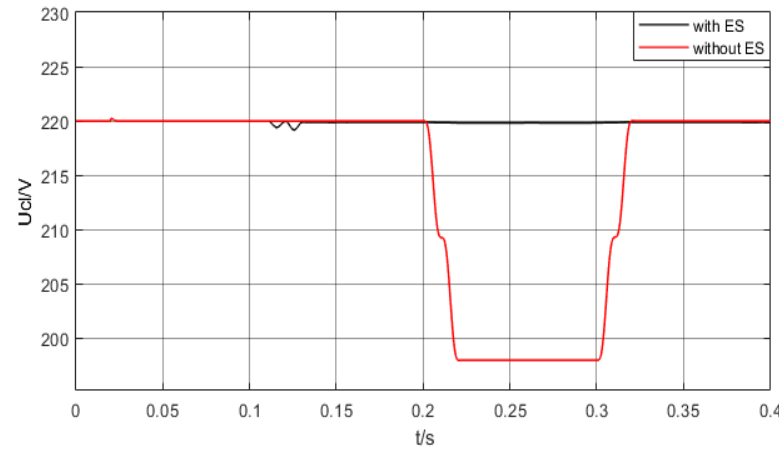

Figure 12. The effective value of $U_{c l}$ at the drop of supplyside voltage

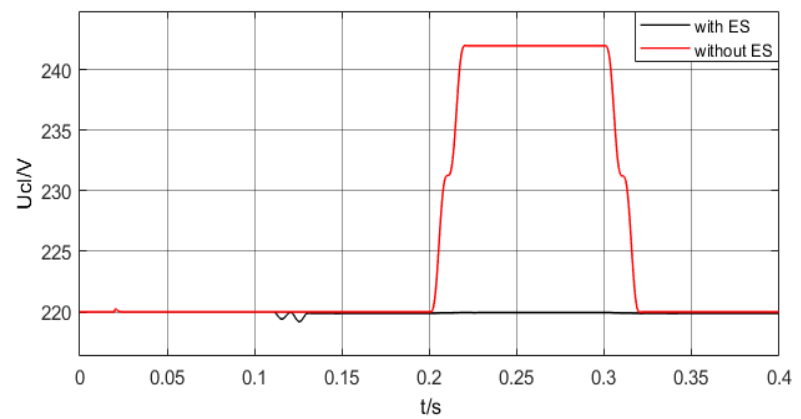

Figure 13. The effective value of $U_{c l}$ at the rise of supplyside voltage

\subsubsection{Simulation at $30 \mathrm{~V}$}

To facilitate the comparison between the simulation results and the experimental results, a MATLAB simulation was carried out at a low voltage $(30 \mathrm{~V})$, with basically the same circuit and controller settings as the simulation at $220 \mathrm{~V}$. The only difference is that the battery voltage Vin was changed from $360 \mathrm{~V}$ to $48 \mathrm{~V}$, and the $U_{\text {ref }}$ was reduced from $220 \mathrm{~V}$ to $30 \mathrm{~V}$. The supply-side voltage was also increased and decreased for the simulation.

Through debugging, it is learned that the CL voltage $U_{c l}$ remained at $30 \mathrm{~V}$, when the ES was not involved in circuit regulation and the supply-side voltage $U_{g}$ was set to $35.69 \mathrm{~V}$. Therefore, the supply-side voltage was decreased and increased by $10 \%$ to $32.04 \mathrm{~V}$ and $39.16 \mathrm{~V}$. The simulation time was set to $0.5 \mathrm{~s}$. The time variations of $U_{g}$ and Tri signal are recorded in Table 4.

Table 4. The time variations of $U_{g}$ and Tri signal

\begin{tabular}{ccc}
\hline Time $/ \mathbf{s}$ & $\mathbf{U g} / \mathbf{V}$ & Tri \\
\hline $0 \sim 0.1$ & 35.69 & 1 \\
$0.1 \sim 0.2$ & 35.69 & 0 \\
$0.2 \sim 0.3$ & 32.04 (drop) & 0 \\
$0.3 \sim 0.4$ & 39.16 (rise) & 0 \\
$0.4 \sim 0.5$ & 35.69 & 0 \\
\hline
\end{tabular}

Figures 14 and 15 compare the amplitudes and effective values of $U_{c l}$ in circuits with and without ES, respectively. In $0 \sim 0.1 \mathrm{~s}$, the supply-side voltage was normal, the Tri signal was 1 , and the switch Q was closed, i.e. the ES was out of service. At $0.1 \mathrm{~s}$, the Tri signal was 0 , and the switch $\mathrm{Q}$ was opened, i.e. the ES was put into use.

As shown in Figure 14, the $U_{c l}$ amplitude in the circuit without ES fluctuated with the dropping or rising voltage, while that in the circuit with ES remained stable. As shown in Figure 15, the circuit with ES achieved a good performance in voltage stabilization. Regardless of voltage drop or voltage rise, the circuit quickly stabilized the effective value of $U_{c l}$ near $30 \mathrm{~V}$. This proves the robustness and adaptability of the ES under the control of FCS-MPC.

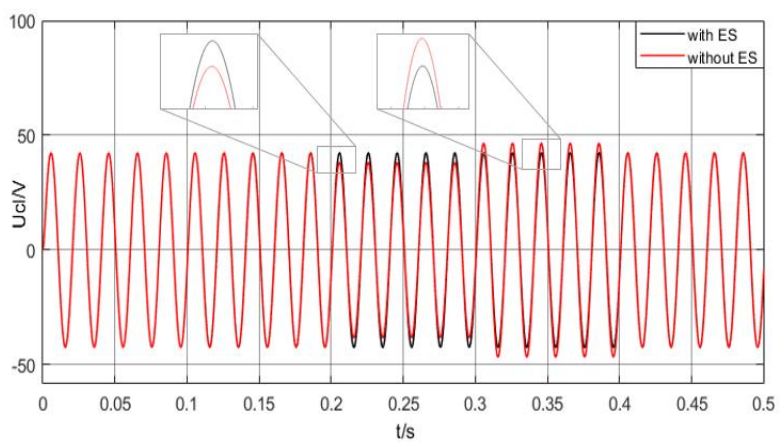

Figure 14. The variations in $U_{c l}$ amplitude at the drop or rise of supply-side voltage

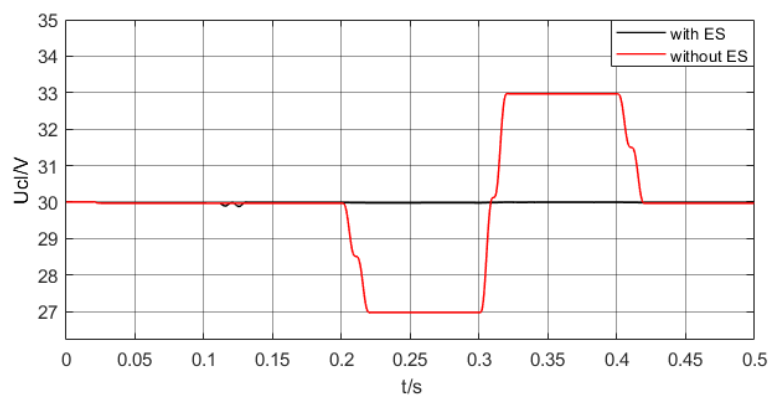

Figure 15. The variations in the effective value of $U_{c l}$ at the drop or rise of supply-side voltage

Figure 16 compares the phases of $U_{c l}$ and $U_{E S}$ at $30 \mathrm{~V}$. In $0 \sim 0.1 \mathrm{~s}$, the Tri signal was 1 , and the switch $\mathrm{Q}$ was closed, i.e. the ES was out of service. In this case, the ES voltage output was 0 . At $0.1 \mathrm{~s}$, the Tri signal was 0 , and the switch $\mathrm{Q}$ was opened, i.e. the ES was put into use. At $0.2 \mathrm{~s}$, the supply-side voltage dropped. In $0.2-0.3 \mathrm{~s}$, the $U_{C}$ lagged $U_{c l}$ by about $90^{\circ}$; the ES worked in capacitive mode under the voltage drop, and injected negative reactive power into the bus to stabilize the voltage across $Z_{c l}$. In $0.3-0.4 \mathrm{~s}$, the supply-side voltage increased, and the grid voltage exceeded the reference value; in this case, the $U_{C}$ led $U_{c l}$ by about $90^{\circ}$; the ES worked in capacitive mode under the voltage rise, and injected positive reactive power into the bus to stabilize the voltage across $Z_{c l}$. The above results further confirm the correctness and effectiveness of our control system.

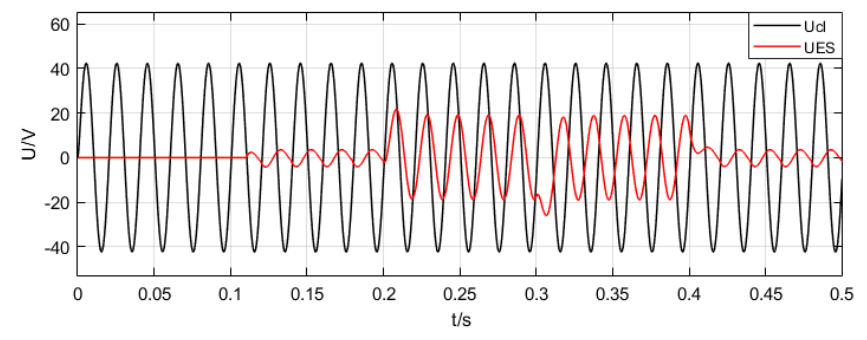

Figure 16. The phase comparison between $U_{c l}$ and $U_{E S}$

\subsection{Physical experiment and result analysis}

Figure 17 illustrates the physical experiment platform. For the safety of physical experiment, four $12 \mathrm{~V} / 24 \mathrm{Ah}$ batteries 
were connected in series to simulate the $48 \mathrm{~V}$ DC power supply. The supply-side power source of the grid was simulated with a voltage regulator, with the effective value of $U_{\text {ref }}$ at $30 \mathrm{~V}$. The control part was simulated with a dSPACE DS1202 controller. The CL and NCL were simulated by adjustable resistors. The controller parameters in dSPACE were kept consistent with those in the simulation at $30 \mathrm{~V}$, except that the step length was adjusted to $5 \mathrm{e}-5 \mathrm{~s}$. To prevent short circuiting, the PWM dead time of the controller output was set to a sampling period.

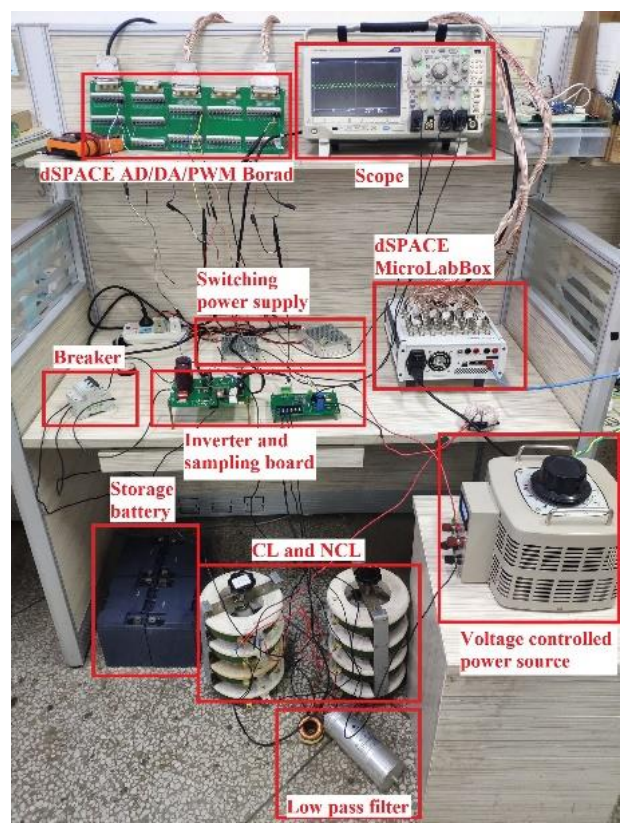

Figure 17. The dSPACE-based ES experimental platform

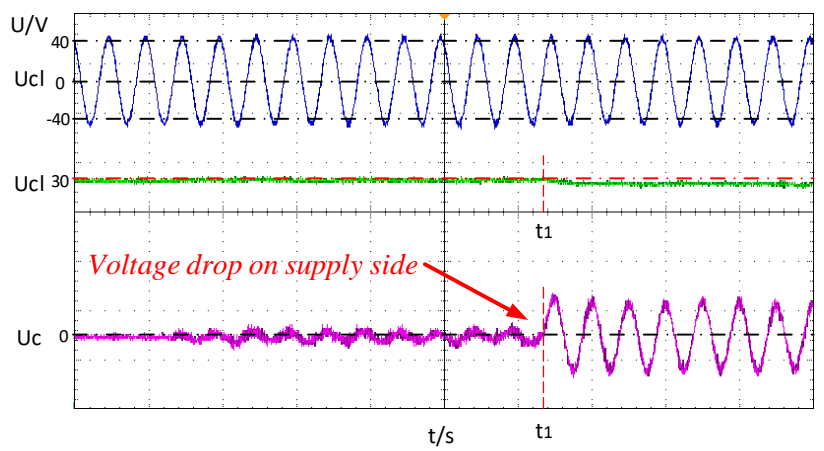

Figure 18. The waveforms of $U_{c l}$ amplitude, $U_{c l}$ effective value, and $U_{C}$ at voltage drop

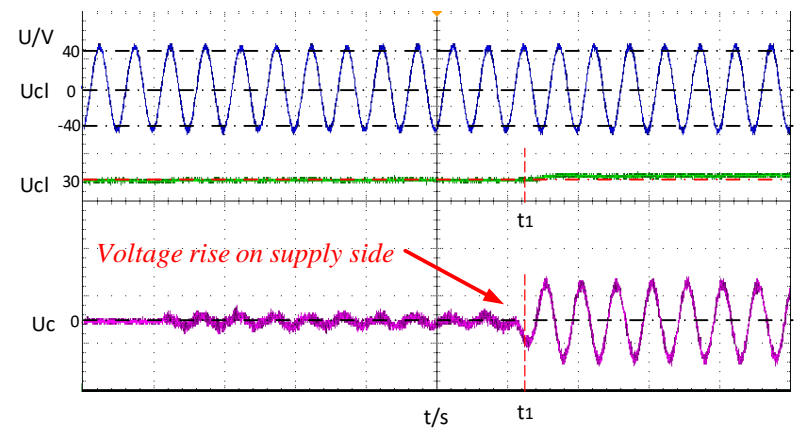

Figure 19. The waveforms of $U_{c l}$ amplitude, $U_{c l}$ effective value, and $U_{C}$ at voltage rise

Figures 18 and 19 compare the $U_{c l}$ amplitudes, $U_{c l}$ effective values, and $U_{C}$ as the supply-side voltage dropped or increased at time $t_{l}$, respectively. It can be seen that $U_{c l}$ amplitude did not fluctuate greatly, while the effective value of $U_{c l}$ stabilized quickly after slight volatility, during the fluctuations of the supply-side voltage.

The ES was put into use after the switch Q was opened, and started to regulate the circuit at time $t_{l}$. During ES regulation, the $U_{C}$ lagged $U_{c l}$ at the drop of supply-side voltage, but led $U_{c l}$ at the increase of supply-side voltage. This is because, the ES worked in capacitive mode under the voltage drop, and injected negative reactive power into the bus to stabilize the voltage across $Z_{c l}$; the ES worked in capacitive mode under the voltage rise, and injected positive reactive power into the bus to stabilize the voltage across $Z_{c l}$.

To clearly observe the ES working states at different voltage fluctuations, the $U_{C}$ phases at the drop and rise of supply-side voltage are compared in Figure 20. As the supply-side voltage started to fluctuate at time $t_{l}$, there was a $180^{\circ}$ difference between the $U_{C}$ phases at the drop and rise of supply-side voltage. The reason is that the $U_{C}$ lagged $U_{c l}$ by $90^{\circ}$ at voltage drop, but led the latter by $90^{\circ}$ at voltage rise. The above analysis shows that the results of physical experiment agree with the simulation results. This further proves the feasibility and effectiveness of our control system.

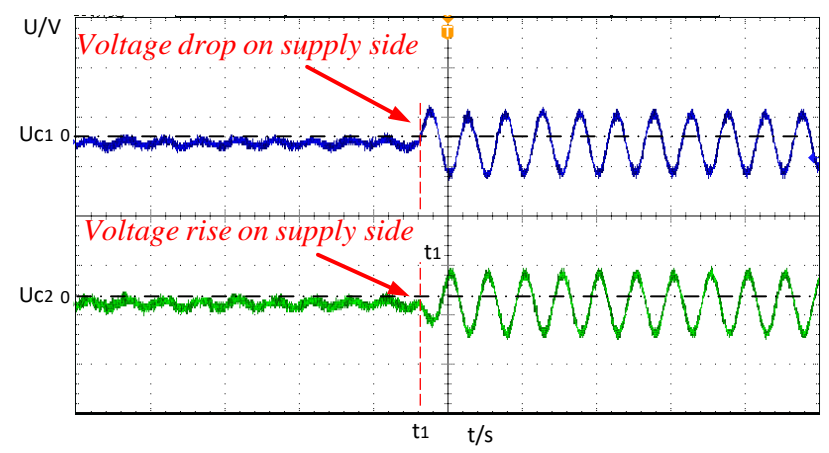

Figure 20. The $U_{C}$ phases at the drop and rise of supply-side voltage

\section{CONCLUSIONS}

With the growing number of nonlinear loads in the circuit, in AC ES control system, the conventional PI controller cannot achieve error-free tracking of the AC reference signal, and the parameter debugging of the traditional linear controller is time-consuming and laborious. In this paper, the FCS-MPC is applied to the ES to solve the problem. After analyzing the model of the ES system, the authors derived the reference voltage of CL, the reference value of ES output voltage, and the predicted value of ES output voltage. On this basis, a control system was designed based on FCS-MPC. Then, the proposed control system was proved effective and correct through simulation on circuits at $220 \mathrm{~V}$ and $30 \mathrm{~V}$. Finally, physical experiment was conducted to manifest the application prospects of the proposed method. The research results provide a good reference for the engineering application of FCS-MPC.

\section{ACKNOWLEDGMENT}

This work was supported in part by Henan mine power electronics device and control innovative technology team 
(Grant No.: CXTD2017085), Science and Technology Planning Project of Henan Province of China (Grant No.: 202102210295) and the Doctoral Scientific Research Foundation of Henan Polytechnic University (Grant No.: B2017-20).

\section{REFERENCES}

[1] Guo, Y., Wang, Q., Yu, J. (2019). Assessment of voltage fluctuations based on wind power fluctuation characteristics. 8th Renewable Power Generation Conference (RPG 2019), Shanghai, China, pp. 1-6. https://doi.org/10.1049/cp.2019.0402

[2] Cheng, M., Zhu, Y. (2014). The state of the art of wind energy conversion systems and technologies: A review. Energy Conversion and Management, 88: 332-347. https://doi.org/10.1016/j.enconman.2014.08.037

[3] Hui, S.Y., Lee, C.K., Wu, F.F. (2012). Electric springsA new smart grid technology. IEEE Transactions on Smart Grid, 3(3): 1552-1561. https://doi.org/10.1109/TSG.2012.2200701

[4] Yan, S., Luo, X., Tan, S.C., Hui, S.R. (2015). Electric springs for improving transient stability of micro-grids in islanding operations. In 2015 IEEE Energy Conversion Congress and Exposition (ECCE), pp. 5843-5850. https://doi.org/10.1109/ECCE.2015.7310480

[5] Che, X., Wei, T., Huo, Q., Jia, D. (2014). A general comparative analysis of static synchronous compensator and electric spring. In 2014 IEEE Conference and Expo Transportation Electrification Asia-Pacific (ITEC AsiaPacific), pp. 1-5. https://doi.org/10.1109/ITECAP.2014.6940991

[6] Mohsenian-Rad, A.H., Leon-Garcia, A. (2010). Optimal residential load control with price prediction in real-time electricity pricing environments. IEEE Transactions on Smart Grid, 1(2): 120-133. https://doi.org/10.1109/TSG.2010.2055903.

[7] Yang, Y., Tan, S.C., Hui, S.Y. (2016). Voltage and frequency control of electric spring based smart loads. In 2016 IEEE Applied Power Electronics Conference and Exposition (APEC), pp. 3481-3487. https://doi.org/10.1109/APEC.2016.7468368

[8] Soni, J., Panda, S.K. (2017). Electric spring for voltage and power stability and power factor correction. IEEE Transactions on Industry Applications, 53(4): 3871-3879. https://doi.org/10.1109/TIA.2017.2681971

[9] Areed, E.F., Abido, M.A. (2015). Design and dynamic analysis of electric spring for voltage regulation in smart grid. In 2015 18th International Conference on Intelligent System Application to Power Systems (ISAP), pp. 1-6. https://doi.org/10.1109/ISAP.2015.7325565

[10] Lee, C.K., Tan, S.C., Wu, F.F., Hui, S.Y.R., Chaudhuri, B. (2013). Use of Hooke's law for stabilizing future smart grid-The electric spring concept. In 2013 IEEE Energy Conversion Congress and Exposition, pp. 5253-5257. https://doi.org/10.1109/ECCE.2013.6647412.

[11] Wang, Q., Cheng, M., Chen, Z., Wang, Z. (2015). Steady-state analysis of electric springs with a novel $\delta$ control. IEEE Transactions on Power Electronics, 30(12): 7159-7169. https://doi.org/10.1109/TPEL.2015.2391278

[12] Zhang, T., Lu, C., Zheng, Z. (2020). Adaptive fuzzy controller for electric spring. European Journal of
Electrical Engineering, 22(3): 233-239. https://doi.org/10.18280/ejee.220304

[13] Yin, F.G., Wang, C. (2019). Review of electric spring: principle, topologies, control and applications. Power System Technology, 43(1): 174-184. https://doi.org/10.13335/j.1000-3673.pst.2018.2040

[14] Richalet, J., Rault, A., Testud, J.L., Papon, J. (1978). Model predictive heuristic control. Automatica (Journal of IFAC), 14(5): 413-428. https://doi.org/10.1016/00051098(78)90001-8

[15] Wang, M., Shi, Y., Shen, M., Wang, H.M., Lu, Y.Y., Qi, M.Y. (2015). Model voltage predictive control for threephase voltage source rectifier. Transactions of China Electro-Technical Society, 30(16): 49-55. https://doi.org/10.3969/j.issn.1000-6753.2015.16.007

[16] Han, J.D., Qi, R., Lei, X.B., Zhang, D.S. (2016). The offline model predictive control for three-phase inverter. Transactions of China Electrotechnical Society, 31(15): 163-169. https://doi.org/10.3969/j.issn.10006753.2016.15.019

[17] Rodriguez, J., Pontt, J., Silva, C., Salgado, M., Rees, S., Ammann, U., Lezana, P., Huerta, R., Cortés, P. (2004). Predictive control of three-phase inverter. Electronics Letters, $\quad 40(9)$ : $561-563$. https://doi.org/10.1109/TIE.2007.899854

[18] Kouro, S., Cortés, P., Vargas, R., Ammann, U., Rodríguez, J. (2008). Model predictive control-A simple and powerful method to control power converters. IEEE Transactions on Industrial Electronics, 56(6): 1826-1838. https://doi.org/10.1109/TIE.2008.2008349

[19] Yang, X.J., Ji, H., Gan, W. (2015). Switching loss optimization based on model predictive control for gridconnected inverter. Electric Power Automation Equipment, 35(8): 84-89. https://doi.org/10.16081/j.issn.1006-6047.2015.08.013

[20] Jin, N., Hu, S., Cui, G., Jiang, S. (2015). Finite state model predictive current control of grid-connected inverters for PV systems. Proc. CSEE, 35(1): 190-196. https://doi.org/10.13334/j.0258-8013.pcsee.2015.S.026

[21] Wang, Q., Deng, F., Cheng, M., Buja, G. (2018). The state of the art of topologies for electric springs. Energies, 11(7): 1724. https://doi.org/10.3390/en11071724

[22] Tan, S.C., Lee, C.K., Hui, S.Y. (2012). General steadystate analysis and control principle of electric springs with active and reactive power compensations. IEEE Transactions on Power Electronics, 28(8): 3958-3969. https://doi.org/10.1109/TPEL.2012.2227823.

[23] Yan, S., Tan, S.C., Lee, C.K., Chaudhuri, B., Hui, S.R. (2014). Electric springs for reducing power imbalance in three-phase power systems. IEEE Transactions on Power Electronics, 30(7): 3601-3609. https://doi.org/10.1109/TPEL.2014.2350001

[24] Zhou, X., Yan, Z. (2019). An application of forward difference method in robust stability of discrete uncertainty system with delays. Advances in Difference Equations, 2019(1): 1-11. https://doi.org/10.1186/s13662-019-1991-x

[25] Zhang, Y., Guo, J., Qiu, B., Li, W. (2019). Zhang neural dynamics approximated by backward difference rules in form of time-delay differential equation. Neural Processing Letters, 50(2): 1735-1753. https://doi.org/10.1007/s1 1063-018-9956-8 